\title{
Sonography for Localization of Central Venous Catheter Location: A Reliable Alternative to Radiography
}

\author{
Yalda Farmani Anoosheh, Saeed Abbasi, Mani Mofidi* \\ Emergency Medicine Management Research Center, Iran University of Medical Sciences, Rasoul Akram Hospital, Tehran, Iran \\ Email address: \\ manimofidi@yahoo.com (M. Mofidi) \\ ${ }^{*}$ Corresponding author \\ To cite this article: \\ Yalda Farmani Anoosheh, Saeed Abbasi, Mani Mofidi. Sonography for Localization of Central Venous Catheter Location: A Reliable \\ Alternative to Radiography. Advances in Surgical Sciences. Vol. 5, No. 6, 2017, pp. 69-72. doi: 10.11648/j.ass.20170506.11
}

Received: September 25, 2017; Accepted: November 11, 2017; Published: December 22, 2017

\begin{abstract}
Introduction: Localization of Control Venous Catheter (CVC) is essential in emergency (ER) and ICU settings; accurately confirming of the location of CVC may enable clinician to minimize the risk of the procedure. Radiography and Sonography are two common procedures, which is used for localization of CVC. Due to volatile hemodynamic nature of patients, it is vital to follow CVC procedure and diagnose possible side effects and complications as soon as possible. Aim of current study is to assess efficacy, practicality and procedure time-curve between two aforementioned methods (Sonography Versus Radiography). After CV line preparation. Methodology: In current cross-sectional study we obtained 201 participants via convenient sampling method from Rasoul-E-Akram Hospital, Haftom-E-Tir Hospital and Firoozgar hospital. Participants were chosen from patients older than 18, who were admitted to ER or ICU in aforementioned hospitals and were candidate for CV line preparation. Following CVC procedure, physicians proceeded with radiography and sonography among all patients and compared approaches with respect to, timing, confirmation of catheter location and post CVC complications. Results: The average time of sonography procedure was estimated roughly 2.08 minutes, in which the maximum time was 5.5 minutes and the minimum time was 1.04 minutes. The standard deviation suggested that the sonography time for most of patients was about 2 minutes. Variance of sonography time among the above population was not reported. The data analysis showed that Chest XRay (CXR) time average for studied patients was about 40.82 minutes, in which the minimum time was 10 minutes and the maximum time was 150.15 minutes. The standard deviation of this variable suggested that variance level and CXR time difference among aforementioned patients were high (about 28.2). Bubble was found in the sonography of 199 cases and just two cases were excluded. According to these results, sensitivity of sonography was $99 \%$, specificity was $100 \%$ and Kappa coefficient between sonography and radiography was $98 \%$. Conclusion: All in all, with respect to high correlation in accurate localization of CVC in both approaches (radiography and sonography) plus competitive advantage of sonography over radiography by maintaining shorter time in confirmation of CVC location, sonography can be discussed as optimum and routine modality with respect catheter location confirmation in emergency settings.
\end{abstract}

Keywords: Catheterization, Central Venous, Interventional, Radiography, Ultrasonography

\section{Introduction}

Central venous catheter (CVC) placement is one of the most prevalent procedures, which take place throughout many major surgeries and in the intensive care unit (ICU) or ER. In the United States, around 5 million CVC is performed with more than $15 \%$ complication rate $[1,2]$. Various factors accounts for complication risks throughout CVC procedure such as operator skill, necessity of placement, among other patient-related considerations such as obesity, prior difficult cannulation and coagulopathy [3, 4]. Despite wide publicity of CVC, this procedure may be accompanied by various complications such as catheter misplacement, pneumothorax, arterial puncture, and hematoma [5-11]. Furthermore, catheter misplacement may lead to more perilous occurrence such as death; hence localization of catheter is essentially 
important procedure $[12,13]$.

To date, radiography is considered gold-standard procedure in confirming localization of $\mathrm{CVC}$ and identifying possible complications. However, in order to follow radiography procedure, patients need to be taken to radiology department, which is time-wasting and in some cases radiography is hazardous for patients such as pregnant women. Furthermore, in high-risk patients time-curve in diagnosis and treatment procedures is vital, thus it is important to follow point of care procedure such as sonography. Various studies have discussed advantages of sonography over radiography $[14,15]$. In a study conducted by Maury and Colleagues (2001), they postulated that ultrasonic diagnosis of catheter misplacement and pneumothorax related to central venous catheterization is a quick and precise approach, which can be easily accomplished by ICU physicians [16]. In another study conducted by Vezzani and Colleagues (2010), they assessed Ultrasound localization of central vein catheter and detection of postprocedural pneumothorax; accordingly they reported that high concordance between ultrasonography plus contrast improved ultrasonography and CXR validates implication of sonography as a reliable approach to confirm the precise localization of the catheter tip and to notice pneumothorax after central venous catheter cannulation [17]. Zanobetti et al. (2011) examined the concordance between chest ultrasonography and chest radiography in patients with dyspnea and they concluded that there is a high concordance rate between radiography and ultrasonography. In cases of dissimilarities between radiography and ultrasonography, latter approach proved to be more accurate in detecting pleural effusion. Hence, according to shorter procedure time in ultrasound, we can discuss that ultrasound can be considered as usual imaging modality for patients with dyspnea [18]. As same as aim of current design, Duran and Colleagues (2015) asked whether ultrasound (US) could more quickly confirm (CVC) position in comparing to CXR in the emergency settings. They reported that Ultrasound could confirm CVC placement and rule out pneumothorax considerably quicker than CXR, accelerating the use of CVCs in the critically ill [19].

Since, we couldn't find similar study in Farsi literature, we aimed to conduct a study Aim to evaluate and compare efficacy, practicality and procedure time-curve between two aforementioned methods (Sonography Versus Radiography) after CV line preparation in emergency department (ED).

\section{Materials and Methods}

In current cross-sectional study we included 201 participants via convenient sampling method from Rasoul-EAkram Hospital, Haftom-E-Tir Hospital and Firoozgar hospital. Participants were chosen from patients older than 18, who were admitted to ER or ICU in aforementioned hospitals and were candidate for CV line preparation. Following CVC procedure, physicians performed radiography and sonography on all patients and compared both approaches with respect to, timing, confirmation of catheter location and possible following complications.

Inclusion Criteria:

1) ICU candidate Patients over 18, who admitted to ER chosen via convenient sampling method

Exclusion Criteria:

2) Pregnant women

3) Patients under 18

We estimated our sample size (201 participants) via following formula:

$$
n=\frac{z^{2}-1-\frac{\alpha}{2} \mathrm{P}(1-p)}{d^{2}}
$$

With respect to assessment of accuracy of sonography cross-tabulation was used.

All participants were informed and explained regarding study procedure and oral consent was acquired from all participants. Throughout this study we precisely followed Helinski ethical principles.

\section{Results}

Two hundred patients were included in current study (male: 129, female: 79). Mean age of male, female and total participants were $59.2 \pm 10,57 \pm 13$ and $58.3 \pm 12$ respectively [Table 1].

According to our obtained data, 168 catheter were positioned in right jugular, 32 in left subclavian and 1 in right subclavian.

Table 1. Demographic Data.

\begin{tabular}{ll}
\hline Total & 201 \\
Male-Age Mean & 72 \\
Age Mean Female & 56.75 \\
Mean Total & 58.32 \\
\hline
\end{tabular}

According to descriptive statistics of sonography result, the average time of sonography procedure was estimated roughly 2.08 minutes, in which the maximum time was 5.5 minutes and the minimum time was 1.04 minutes. The standard deviation suggested that the sonography time for most of patients was about 2 minutes [Table 2].

Table 2. Sonography Duration Statistics.

\begin{tabular}{llllll}
\hline Variable & Number & Minimum & Maximum & Mean & SD \\
\hline Sonography Duration (minutes) & 201 & 1.04 & 5.5 & 2.08 & 0.77731 \\
CXR Duration (minutes) & 200 & 10 & $150 / 15$ & $40 / 82$ & $28 / 2053$ \\
\hline
\end{tabular}


which the minimum time was 10 minutes and the maximum time was 150.15 minutes. The standard deviation of this variable suggested that variance level and CXR time difference among aforementioned patients were high (about 28.2 minutes more or less) [Table 2].

As inferred from comparison of data in Table 1 and 2, duration of CXR in comparison to sonography is significantly higher and CXR procedure takes more time comparing to sonography. In order to compare relationship between CXR and sonography duration Pearson correlation test was used. In result, no meaningful relationship was found between two aforementioned approaches.

Bubble was found in the sonography of 199 cases and just two cases were excluded and CVC was not spotted via sonograph. According to these results, sensitivity of sonography was $99 \%$, specificity was $100 \%$ and Kappa coefficient between sonography and radiography was $98 \%$ [Table 3].

Table 3. Symmetric Measures.

\begin{tabular}{|c|c|c|c|c|c|}
\hline & & Value & Asymp. Std. Error ${ }^{a}$ & Approx. $\mathbf{T}^{\mathrm{b}}$ & Approx. Sig. \\
\hline Measure of Agreement & Kappa & .980 & .014 & 13.895 & .000 \\
\hline $\mathrm{N}$ of Valid Cases & & 201 & & & \\
\hline
\end{tabular}

\section{Discussion}

According to results of current study, it appears plausible that with respect to high correlation in accurate localization of CVC in both approaches (radiography and sonography) plus competitive advantage of sonography over radiography by maintaining shorter time in confirmation of CVC location, sonography can be considered as optimum modality with respect catheter location confirmation in ER.

Results of current study are consistent with the result of the study conducted by Mauri et al. (2001). However, in their study they had 85 participants and in our study we had 201 participants. Level of post CVC complications was more in aforementioned study comparing to current study. Furthermore, Vezzani and Colleagues (2010), found high correlation $(\mathrm{kappa}=88 \%)$ between sonography and radiography. In current study we found correlation kappa $=98 \%$ between two aforementioned approaches. This difference may stems from different in sample size in our study and Vezzani's study (101 participants VS 201 participants). Nonetheless, both of studies show high correlation in confirmation of localization of CVC.

In another study Matsushima and Frankel (2010) hypothesized hypothesized whether more precise ultrasound surveillance procedure including thoracic, vascular, and cardiac views-the CVC sono-would hinder the necessity for chest radiography or not. In consistent with the results of current study they concluded that CVC sonography could eliminate the call for CXR in most patients after CVC/PICC insertion, saving time and money [20].

Finally, in a study conducted by Zanobetti and Colleagues (2012), they aimed to verify optimal central venous catheter placement in the emergency department via comparing ultrasonography and chest CXR. Consistent with the result of current study they concluded that there is high correlation between aforementioned modalities in identifying possible malpositioning of the catheter. Furthermore, less time is required to perform ultrasonography comparing to CXR [21].

According to our study results and various literatures, sonography can be considered goal standard approach and substitute modality in confirmation of CVC placements in ER.

\section{Limitation}

It is advisable to conduct current study with bigger statistical sample. Furthermore, our participants were not matched according to height, BMI and etc. ER chaotic environment indirectly would have influenced timing procedure of CXR and sonography. Skillfulness of clinicians is essential in timing of the procedure and should be noted and controlled during study.

\section{Acknowledgements}

We would like to express our gratitude to all participants in current study.

\section{References}

[1] Sznajder JI, Zveibil FR, Bitterman H, Weiner P, Bursztein S. Central vein catheterization: Failure and complication rates by three percutaneous approaches. Arch Intern Med. 1986;146:259-61. [PubMed]

[2] Defalque RJ. Percutaneous catheterization of the internal jugular vein. Anesth Analg. 1974;53:116-21.[PubMed]

[3] Goldfarb G, Lebrec D. Percutaneous cannulation of the internal jugular vein in patients with coagulopathies: An experience based on 1,000 attempts. Anesthesiology. 1982;56:321-3. [PubMed]

[4] Mansfield PF, Hohn DC, Fornage BD, Gregurich MA, Ota DM. Complications and failures of subclavianvein catheterization. N Engl J Med. 1994;331:1735-8. [PubMed]

[5] Ruesch S, Walder B, Tramer MR. Complications of central venous catheters: internal jugular versus subclavian access--a systematic review. Crit Care Med. 2002;30(2):454-60. [PubMed]

[6] Aminnejad R, Razavi SS, Mohajerani SA, Mahdavi SA. Subclavian Vein Cannulation Success Rate in Neonates and Children. Anesth Pain Med. 2015;5(3):24156. doi: 10.5812/aapm.24156v2.[PMC free article] [PubMed] [Cross Ref] 
[7] Moeinipour AA, Amouzeshi A, Joudi M, Fathi M, Jahanbakhsh S, Hafez S, et al. A rare central venous catheter malposition: a case report. Anesth Pain Med. 2014;4(1):16049. doi: 10.5812/aapm.16049.[PMC free article] [PubMed] [Cross Ref]

[8] Lie J, Yadagiri M, Aspinall C. Letter to editor on a rare central venous catheter malposition. Anesth Pain Med. 2014;4(4):20019. doi: 10.5812/aapm.20019. [PMC free article] [PubMed] [Cross Ref]

[9] Fathi M, Izanloo A, Jahanbakhsh S, Taghavi Gilani M, Majidzadeh A, Sabri Benhang A, et al. Central Venous Cannulation of the Internal Jugular Vein Using UltrasoundGuided and Anatomical Landmark Techniques. Anesth Pain Med. In Press;In Press:35803. [PMC free article] [PubMed]

[10] Lentz RJ, Sandler KL, Miller AN. Cannulation of the Internal Thoracic Artery with a Central Venous Catheter. Am J Respir Crit Care Med. 2016;193(5):9-11. doi: 10.1164/rccm.2015091748IM. [PubMed] [Cross Ref]

[11] Shah PN, Kane D, Appukutty J. Depth of central venous catheterization by intracardiac electrocardiogram in adults. Anesth Pain Med. 2013;2(3):111-4. doi: 10.5812/aapm.7557.[PMC free article] [PubMed] [Cross Ref]

[12] Orme RM, McSwiney MM, Chamberlain-Webber RF. Fatal cardiac tamponade as a result of a peripherally inserted central venous catheter: a case report and review of the literature. $\mathrm{Br} \mathrm{J}$ Anaesth. 2007;99(3):384-8. doi: 10.1093/bja/aem181. [PubMed] [Cross Ref]

[13] Collier PE, Blocker SH, Graff DM, Doyle P. Cardiac tamponade from central venous catheters. Am J Surg. 1998;176(2):212-4. [PubMed]

[14] Xirouchaki N, Magkanas E, Vaporidi K, et al. Lung ultrasound in critically ill patients: comparison with bedside chest radiography. Intensive Care Med. 2011;37:14,88-93.
[15] Lichtenstein DA, Meziere GA. Relevance of lung ultrasound in the diagnosis of acute respiratory failure: the BLUE protocol. Chest. 2008;134:117-25.

[16] Maury E, Guglielminotti J, Alzieu M, Guidet B, Offenstadt G. (2001). Ultrasonic examination: an alternative to chest radiography after central venous catheter insertion?. Available: https://www.ncbi.nlm.nih.gov/pubmed/11500340. Last accessed October-2017.

[17] Vezzani A, Brusasco C, Palermo S, Ultrasound localization of central vein catheter and detection of postprocedural pneumothorax: an alternative to chest radiography. Crit Care Med. 2010 Feb;38(2):533-8.

[18] Zanobetti M, Poggioni C, Pini R. (2011). Can chest ultrasonography replace standard chest radiography for evaluation of acute dyspnea in the ED? Available: https://www.ncbi.nlm.nih.gov/pubmed/20947649. Last accessed October-2017.

[19] Duran-Gehring PE, Guirgis FW, McKee KC, The bubble study: ultrasound confirmation of central venous catheter placement. Am J Emerg Med. 2015 Mar;33(3):315-9.

[20] Matsushima K, Frankel HL. (2010). Bedside ultrasound can safely eliminate the need for chest radiographs after central venous catheter placement: CVC sono in the surgical ICU (SICU). Available: https://www.ncbi.nlm.nih.gov/pubmed/20599208. Last accessed October-2017.

[21] Zanobetti M, Coppa A, Bulletti F, Piazza S, Nazerian P, Conti A, Innocenti F, Ponchietti S, Bigiarini S, Guzzo A, Poggioni C, Taglia BD, Mariannini Y, Pini R. (2012). Verification of correct central venous catheter placement in the emergency department: comparison between ultrasonography and chest radiography. Available: https://www.ncbi.nlm.nih.gov/pubmed/23242559. Last accessed October-2017. 